\title{
Are the stabilities of stablecoins connected?
}

\author{
Binh Nguyen Thanh ${ }^{1}$ Thai Nguyen Vu Hong ${ }^{1}$ Huy Pham ${ }^{1}$. \\ Thanh Nguyen Cong ${ }^{2}$. Thu Pham Thi Anh'
}

Received: 3 August 2021 / Revised: 4 January 2022 / Accepted: 10 January 2022

(c) The Author(s) 2022

\begin{abstract}
This study analyzes the interconnection of the stability of prominent stablecoins including Tether (USDT), USD Coin (USDC), Paxos Standard (PAX), TrueUSD (TUSD) and DAI from 23/11/2019 to $1 / 04 / 2021$. We find (i) market price fluctuations vary across stablecoins, (ii) instabilities in USDT and USDC significantly drive those of other stablecoins but less convincing evidence for the reverse impact, and (iii) that increases (decreases) in USDT market prices significantly depress (raise) the market prices of the other stablecoins. Interestingly, the algorithmic stablecoin, DAI, is found to be less stable than its USD-backed counterparts.
\end{abstract}

Keywords Cryptocurrency · Stablecoin · Vector Autoregression

Huy Pham

huy.phamnguyenanh@rmit.edu.vn

Binh Nguyen Thanh

binh.nguyenthanh@rmit.edu.vn

Thai Nguyen Vu Hong

thai.nguyenvuhong@rmit.edu.vn

Thanh Nguyen Cong

thanhnc@uef.edu.vn

Thu Pham Thi Anh

elliepham.finance@gmail.com

1 School of Business and Management, RMIT University, Vietnam Campus, 702 Nguyen Van Linh, District 7, Ho Chi Minh City, Vietnam

2 School of Finance - Commerce, Ho Chi Minh City University of Economics and Finance (UEF), 141-145 Dien Bien Phu, Ward 15, Binh Thanh District, HCM City, Vietnam 


\section{Introduction}

Cryptocurrencies have attracted a lot of attention from academia in the last years with a large part of the literature focusing on the return and volatility of cryptocurrencies (e.g., Dyhrberg, 2016; Demir et al., 2018; Nguyen et al., 2019 and Nguyen et al., 2019). Other works investigate Bitcoin's suitability to act as money and most studies point out that Bitcoin has not been primarily used as a medium of exchange. Baur et al. (2018) find a small proportion of users who treat Bitcoin as a medium of exchange and conclude that investors and a large percentage of users consider Bitcoin as a speculative investment instead of an alternative currency or a medium of exchange. According to Beigel (2020), Bitcoin is not universally accepted as a medium of exchange although several multinational corporations accept Bitcoin payments. In addition, de la Horra, de la Fuente and Perote (2020) and Hazlett and Luther (2019) show that Bitcoin transactions only take up a negligible proportion of all global transactions (including cash and non-cash) and Bitcoin is only used as a medium of exchange in a relatively small domain. The biggest problem with Bitcoin or most cryptocurrencies lies in their high volatility due to the inability to place a correct valuation (Smales, 2019). If goods and services are priced in Bitcoin, businesses would incur high price-changing costs due to the high volatility of Bitcoin (de la Horra et al., 2020). This highly volatile nature of most cryptocurrencies might result in the fluctuation of purchasing power relative to the supply and prices of goods denominated in other currencies. Given the high volatility of cryptocurrencies, stablecoins have been introduced to the market and are expected to have stable value as they are pegged to another asset (Allen et al., 2021).

Given the exponential rise of the cryptocurrency market, a new strand of literature has investigated the role of stablecoins in the cryptocurrency market. Baur and Hoang (2020), for instance, indicate that Bitcoin investors use stablecoins when Bitcoin exhibits an extreme negative decrease in price and their results show that stablecoins might not live up to their name and fail to fulfil their initial purposes (i.e. being a stable cryptocurrency) because they provide a strong crypto safe haven against Bitcoin. Similarly, Bullmann et al. (2019) find a trade-off between the innovations of the stabilization mechanism of a stablecoin and its ability to maintain a stable market value. Regardless of the potential importance of stability in the cryptocurrency market, a few studies have examined if stablecoins are truly stable. With the regard to the stability of stablecoins, Hoang and Baur (2020) identify Bitcoin as a source of excess volatility in stablecoins and also provide evidence that stablecoins contribute to the excess volatility of Bitcoin. In addition, Grobys (2021) find the spillover effects of Bitcoin volatility on stablecoins. We extend the scope of the studies of Hoang and Baur (2020) and Grobys (2021) and ask if there exists a volatility spillover among stablecoins. The connectedness in the cryptocurrency market has been extensively studied for traditional cryptocurrencies, but not for stablecoins, so our study attempts to fill this gap. The literature on the correlation among traditional cryptocurrencies largely agrees that Bitcoin is the center of the connectedness. For example, Moratis (2021) examines the pairwise directional spillover shocks for the 30 largest cryptocurrencies (including 1 stablecoin) and finds that Bitcoin dominates others in the risk transmission process. In addition, by investigating the tail-risk interdependence 
among 23 cryptocurrencies, $\mathrm{Xu}$ et al. (2021) document the increasing connectedness of traditional cryptocurrencies and point out Bitcoin as the largest systemic risk receiver. By examining the return connectedness of seven largest traditional cryptocurrencies, Bouri et al. (2021) find that connectedness measures are higher in the left and right tails than those in the mean and median, indicating that return shock spillover is more intense during extreme events. Ferreira et al. (2020) examine the serial correlation structure of six traditional cryptocurrencies and document that the serial correlations become insignificant during the first 30 days, indicating the relatively faster decay compared to stock indices. These previous studies focus only on traditional cryptocurrencies, the volatility spillover among stablecoins remains unexplained in the literature.

Considering the increasing adoption of stablecoins by the market participants, it is crucial to expand the knowledge of stablecoins as most studies only analyze the stablecoins' reaction to extreme movement in non-stable coins' price movement or the interaction between stablecoins and Bitcoin while neglecting the interconnection of the stability of stablecoins themselves. The current study seeks to contribute to the literature by analyzing the relationship of the stabilities of the largest five stablecoins. In this regard, we argue that signaling theory plays a significant role in explaining the interrelation between stablecoins. As cryptocurrency market and stablecoins are in their early stage of development, the tech-savvy in this market may create information asymmetry to investors for their trading decisions. This is in line with the arguments from Philippi et al. (2021) that the technological nature of Initial Coin Offerings (ICOs) is the source of information asymmetry, and hence, publishing a source code is an effective signal vehicle for ICO fundraising success. In the context of stablecoins, we argue that the price movement of large-cap stablecoins can be interpreted as signals on trading opportunities for smaller-cap stablecoins by investors. Therefore, large-cap stablecoins tend to drive smaller ones. The findings are expected to show how larger stablecoin price changes influence the prices of lower market cap stable coins because stablecoins users can easily switch between different types of stablecoins in the cryptocurrency market.

The rest of the paper is organized as follows. Section 2 describes the data and the econometric model; Sect. 3 presents the estimation results and Sect. 4 concludes the paper.

\section{Data and econometric model}

We use a sample of five largest stablecoins in terms of market capitalization including Tether (USDT), USD Coin (USDC), Paxos Standard (PAX), TrueUSD (TUSD) and Multi Collateral DAI (DAI) from 23/11/2019 to 1/04/2021. The stablecoins have the nominal value of $\$ 1$ and the daily data are fetched from coinmarketcap.com. We select this sample period due to the data availability whereby Multi Collateral DAI is only available from 23/11/2019. According to Coinmarketcap, the price of any cryptoassets is a volume-weighted average of market pair prices in several exchanges such as Binance or Huobi. DAI is the only algorithmic stablecoin in our sample that is created through users using Ether and other Ethereum-based cryptocurrencies as 
collateral to effectively borrow DAI, whereas the other stablecoins are backed by USD. The cryptocurrency market has several distinct features compared to the traditional financial market. Cryptocurrencies are traded 24/7 and the crypto exchanges are effectively never closed. There are cryptocurrency exchanges in almost every country in the world allowing investors to invest with any fiat currency. Those features make the cryptocurrency market a global market and allow traders to react to new information rapidly.

We use the two following measures of the stability of stablecoins:

- Measure (1): The deviation of the stablecoin's market price from its nominal value.

$$
M 1 \_i_{t}=\text { ClosePrice } \text { Pl }-1
$$

where Close Price $_{t}$ denotes the end of the day price of the respective stablecoin $i$ on day $t$, respectively. Measure (1) captures how close the market prices of the stablecoins stick to their nominal values; the closer to zero, the better the stablecoin's behavior sticking to its nominal value. A positive (negative) Measure (1) means that investors have paid a higher (lower) price for the stablecoin than its nominal value. An increase in this Measure (1) means that the market price of stablecoin is increasing.

- Measure (2): The realized volatility measure inspired by Parkinson (1980) HighLow measure and utilized by Bollen and Inder (2002).

$$
M 2_{-} i_{t}=\sqrt{\frac{\left(\ln H i g h_{t}-\ln L o w_{t}\right)^{2}}{4 \ln 2}},
$$

where High and Low denote the highest and lowest price for cryptocurrency $i$ on day $\mathrm{t}$, respectively.

Fig. 1 Stability Measure (1) of the stablecoins over time, 23/11/2019- $1 / 04 / 2021$

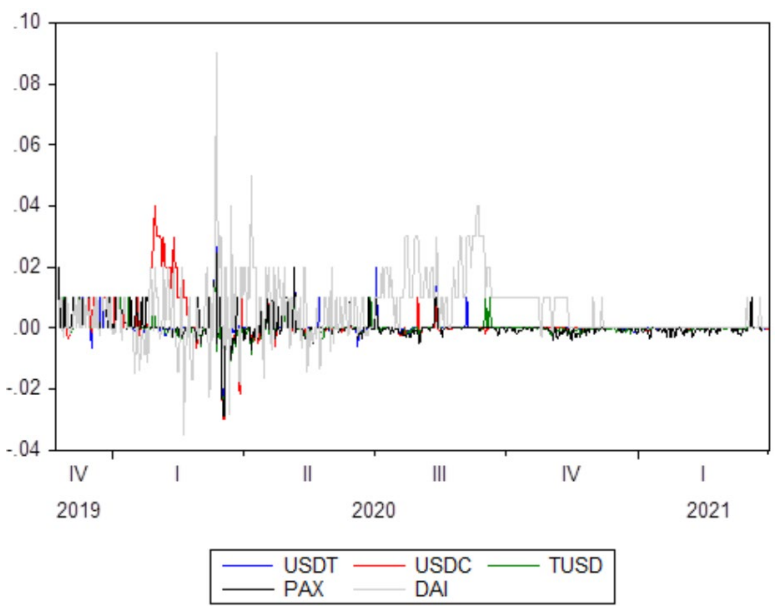


Figure 1 displays Measure (1), the deviation of stablecoins market prices from their nominal values, and Fig. 2 illustrates Measure (2), the realized volatility measure of the stablecoins over the sample period. Although the market prices of stablecoins are moderately stable and exhibit mean-reverting processes, there are numerous episodes associated with great deviations from the nominal price and high market price volatility. For example, the highly volatile market prices of stablecoins can be observed around March 2020.

Table 1 captures the descriptive statistics of those two measures of stablecoin market price stability. The widest range of Measure (1) can be observed for DAI with market prices exceeding and undershooting the nominal price by up to $\$ 0.09$ (or $9 \%^{1}$ ) and $\$ 0.035$ (or 3.5\%), respectively, translating to a range of $\$ 0.125$ or $12.5 \%$. The volatility DAI's Measure (1) is with $\$ 0.0107$ (or 1.07\%) the highest among all stablecoins. Moreover, the algorithmic stablecoin DAI also displays the highest average Measure (2) suggesting that its value is overall less stable compared to its USD-backed counterparts. This could be interpreted as higher (perceived) risk of DAI compared to the USD-backed stablecoins, since DAI is algorithmically backed by Ethereum and Ethereum-based cryptocurrencies which are more volatile in value than USD. Interestingly, the Ethereum-based USDC has been increasingly used as collateral to mint DAI in recent times even surpassing the amount of Ethereum that is being used as collateral to create DAI at times. Moreover, the deviation of the market price of a stablecoin from its nominal value could reflect the excess or under demand for the respective stablecoin compared to its supply. Particularly, a deviation to the downside of the market price could reflect a lower degree of trust to the sufficient backing of the stablecoin (e.g., Griffin and Shams (2020) find that USDT may not being adequately backed by USD). Table 2 shows the average market capitalization and daily trading volume for the cryptocurrencies in our sample. While the market

Fig. 2 Stability Measure (2) of the stablecoins over time, 23/11/2019-01/04/2021

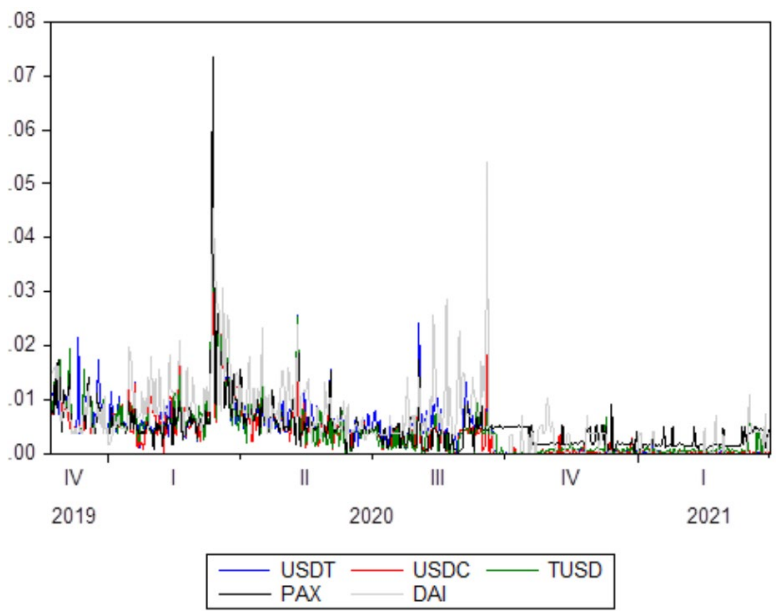

${ }^{1} \$ 0.09$ deviation translates to $9 \%$ deviation, since the nominal price of DAI (and all other Stablecoins in the paper) is $\$ 1$. 
Table 1 Descriptive statistics

\begin{tabular}{llllll}
\hline Variable & Obs & Mean & Std.Dev. & Min & Max \\
\hline BTC & 496 & 0.017307 & 0.014836 & 0.003001 & 0.176525 \\
M1_USDT & 496 & 0.000702 & 0.004368 & -0.0258 & 0.05 \\
M1_USDC & 496 & 0.001462 & 0.00644 & -0.0299 & 0.04 \\
M1_PAX & 496 & 0.000395 & 0.004725 & -0.0292 & 0.05 \\
M1_TUSD & 496 & 0.000341 & 0.004186 & -0.0291 & 0.04 \\
M1_DAI & 496 & 0.005683 & 0.010775 & -0.0352 & 0.09 \\
M2_USDT & 496 & 0.004255 & 0.005437 & 0 & 0.065959 \\
M2_USDC & 496 & 0.003611 & 0.004750 & 0 & 0.047501 \\
M2_PAX & 496 & 0.004685 & 0.004995 & 0 & 0.073472 \\
M2_TUSD & 496 & 0.003965 & 0.005107 & 0 & 0.055301 \\
M2_DAI & 496 & 0.006084 & 0.006488 & 0 & 0.05414 \\
\hline The table shows the & descriptive & statistics of the stability Measure (1) and & Measure (2), \\
23/12/2019-01/04/2021 & & &
\end{tabular}

price of the various stablecoins are often not exactly $\$ 1$, substantial deviations from the nominal values can be observed for limited time periods only.

Table 3 shows the correlation coefficients of the stability measures of the stablecoins. Interestingly, M1_USDT is negatively correlated with all other stablecoins' Measure (1) whereas the correlation turns positive in the other stablecoins, indicating that the other stablecoins might potentially act as substitutes for USDT. All correlations of the stability Measure (2) are statistically significant and positive, suggesting that the market price volatilities of the stablecoins are mainly positively related.

To further analyze the dynamic relationship of the stablecoin stability Measure (1) and Measure (2), we utilize a Vector Autoregression (VAR) model to estimate the impulse responses of each stablecoin's stability measure to shocks in the other stablecoins. A representation of the $\operatorname{VAR}(p)$ is

$$
Y_{t}=\sum_{i=1}^{p} A_{i} Y_{t-i}+\epsilon_{t}(1)
$$

where $A$ contains the coefficient matrix, $\epsilon_{t}$ is the vector of errors and $Y_{t}$ is the vector containing all endogenous variables including Measure (2) of Bitcoin and the measures of stability of the stablecoins. $Y_{t}$ can be represented as follows.

Table 2 Average trading volume and market capitalization

\begin{tabular}{llll}
\hline Variable & $\begin{array}{l}\text { Average 24-hour } \\
\text { Trading Volume (in USD) }\end{array}$ & $\begin{array}{l}\text { Average Market } \\
\text { Capitalization (in USD) }\end{array}$ & Obs \\
\hline Bitcoin & 38,465 Million & 317,074 Million & 496 \\
Tether & 54,935 Million & 13,813 Million & 496 \\
USD Coin & 759 Million & 2421 Million & 496 \\
True USD & 263 Million & 237 Million & 496 \\
Paxos Standard & 289 Million & 290 Million & 496 \\
Single_Collateral DAI & 112 Million & 687 Million & 496 \\
\hline
\end{tabular}

The table shows the average 24-hour trading volume and the average market capitalization of the stablecoins, 23/11/2019-01/04/2021 


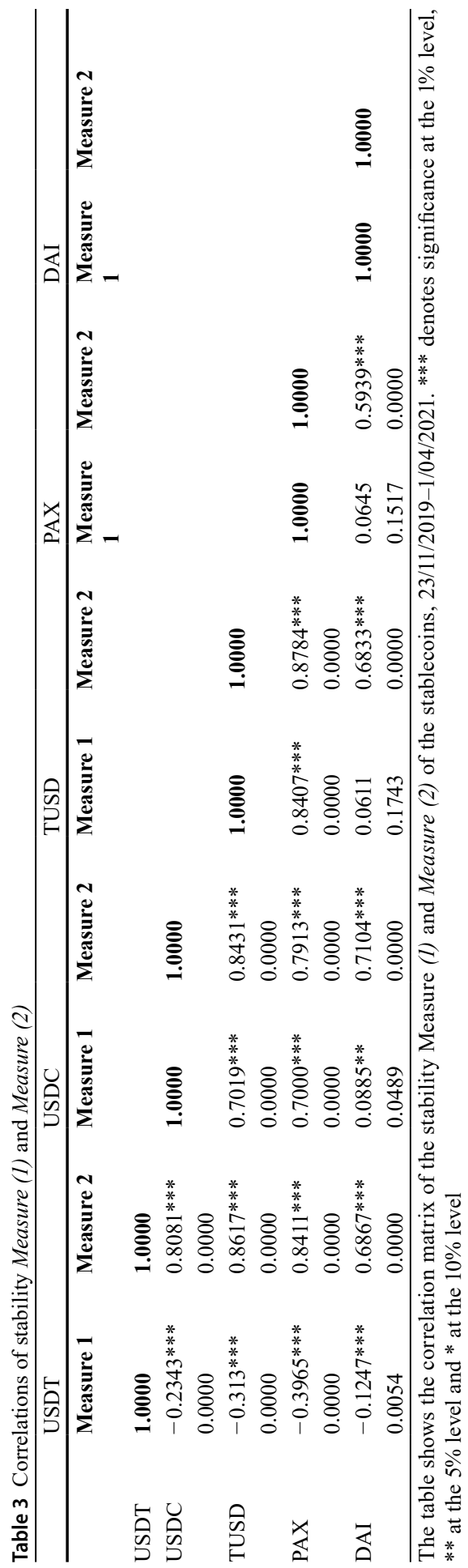




$$
\left(\begin{array}{c}
{\text { BitcoinMeasure }(2)_{t}} \\
\text { Tetherstabilitymeasure }_{t} \\
\text { USDCoinstabilitymeasure }_{t} \\
\text { TrueUSDstabilitymeasure }_{t} \\
\text { PaxosStandardstabilitymeasure }_{t} \\
\text { DAIstabilitymeasure }_{t}
\end{array}\right)
$$

We estimate Eq. (3) for Measure (1) and Measure (2) separately. We include the realized volatility Measure (2) of Bitcoin price to test whether Bitcoin price volatility also affects the stability of the stablecoins. We select the optimal lag length based on the recommendation of the AIC, SC, HQ criteria and sequential modified LR test statistic to use five lags. Note that the stability measures for all stablecoins are found to be stationary ${ }^{2}$.

\section{Estimation results and discussion}

Figure 3 shows the impulse responses of the stability Measure (1) of the stablecoins to shocks in stability Measure (1) of all cryptocurrencies. An increase in USDT significantly decreases all other stablecoins and further suggests that the smaller-cap stablecoins are used as substitutes for Tether. Interestingly, an increase (decrease) in USDC (the second-largest stablecoin in terms of market capitalization) also significantly increases (decreases) all other smaller-cap stablecoins including PAX, TUSD and DAI, suggesting that if the market price of USDC goes up, the market price for PAX, TUSD and DAI increases as well. We find less statistically significant evidence to prove that Measure (1) of the smaller-cap stablecoins drive those of the larger-cap stablecoins such as USDT and USD Coin. Moreover, an increase in Bitcoin Measure

Fig. 3 VAR impulse responses of the stablecoin stability Measure (1) - The deviation of the market price from the nominal price $\$ 1,23 / 11 / 2019-1 / 4 / 2021$

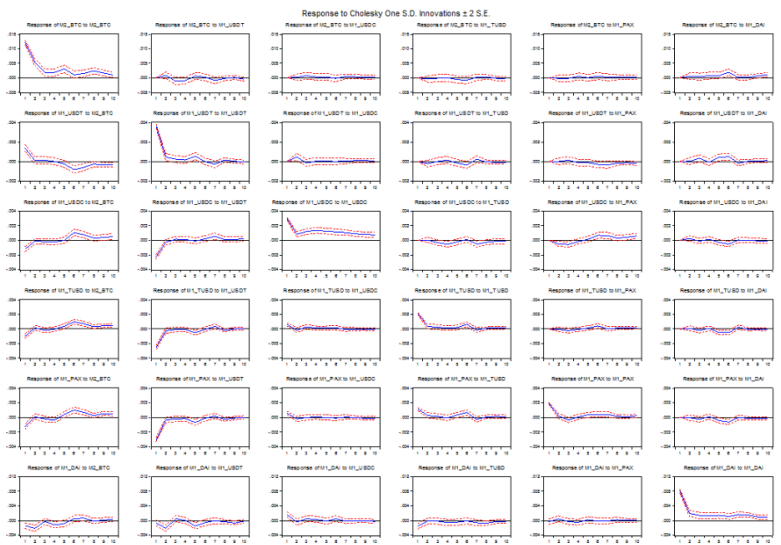

\footnotetext{
${ }^{2}$ Data is available upon request.
} 
(2) raises the price of USDT which indicates that there may be a higher demand for USDT when the Bitcoin price is volatile.

Figure 4 shows the VAR impulse response estimations for the stability Measure (2) to shocks in the stability Measure (2) of all cryptocurrencies. An increase in USDT market price volatility significantly raises the market price volatility of the remaining stablecoins, whereas the reverse effects are mostly not significant. Similarly, the USD Coin market price volatility significantly drives the market price volatility of the smaller market-cap coins, such as TUSD, PAX and DAI. We find less statistically significant evidence for the small-cap coins affecting the large-cap cryptocurrencies. The findings further suggest that when the USDT price fluctuates, investors allocate their capital to smaller capitalization stablecoins. These results support our arguments the price movement of large-cap stablecoins can send some signals to investors on trading opportunities for small-cap stablecoins.

Furthermore, an increase in the Bitcoin price volatility leads to a surge in Measure (2) of all stablecoins, indicating that Bitcoin price volatility is an important driver of stablecoin price volatilities. This finding is consistent with those of Lyons and Viswanath-Natraj (2020) who find USDT is traded at a premium when there is an increased risk in cryptocurrency markets. For robustness check, we estimate various specifications of Structural Vector Autoregression Models (SVAR) to impose different order restrictions on the contemporaneous relationships of the stablecoins, employ different lag lengths in the VAR and SVAR estimation, and find consistent results.

\section{Conclusions}

This study sheds light on the stability of the five largest stablecoins in terms of market capitalization and their interconnection. Besides the counterparty risk of privately issued stablecoins as pointed out by Griffin and Shams (2020), any instability of stablecoins can be a significant obstacle for users who want to use a digital cryptocurrency-version of the US dollar to settle transactions. We find occasional periods

Fig. 4 VAR impulse responses of the stablecoin stability $\mathrm{Mea}$ sure (2)_-The realized volatility of the stablecoin market price, $23 / 112,019-1 / 04 / 2021$
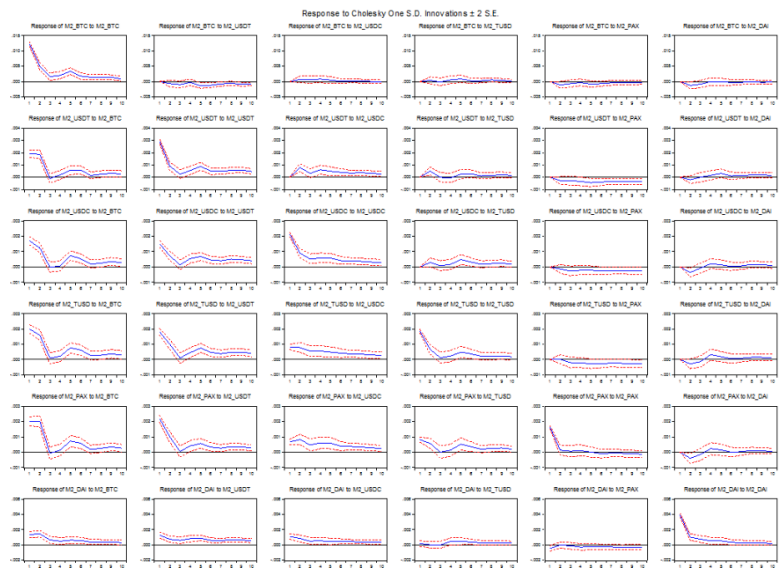

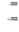

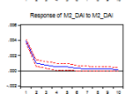


in which the market prices of stablecoins substantially deviate from their nominal values that question the level of the self-acclaimed stability feature of the stablecoins in those periods. A Central Bank Digital Currency that is issued directly by the central banks could potentially eliminate those issues and provide a stable medium of exchange for settling transactions as discussed by Eichengreen (2019) and Hairudin et al. (2020). Our results also show that algorithmic stablecoins perform worse compared to privately issued stablecoins.

In addition, the study reveals strong interconnections of the stability of the stablecoins, with USDT and USD Coin driving the stability of their low-cap counterparts, but less statistically significant evidence for the reverse impact. This finding provides an interesting fact that higher market-cap stablecoins drive the smaller market-cap counterparts. Due to the limited number of stablecoins included in this study, this observation should be interpreted with caution, and future research should extend the sample with a significant number of coins for statistical validation. The findings also suggest that users could easily substitute stablecoins when they have the same nominal value, particularly, if the market price of a stablecoin is higher than its nominal price. The estimation results also suggest that USDC, TUSD, PAX and Dai are used as substitutes for USDT.

Authors contributions BNT is the lead author and has developed the idea of the study as well as has drafted the work. TNVH has substantively revised the paper. HP has provided the literature review and has reshaped the paper. TNC has collected and analyzed the data. TPTA has visually depicted and interpreted the data.

Funding For the study, the 2019 RMIT Vietnam Internal Research Grant was utilized to hire a Research Assistant who collected the data and conducted the estimations.

\section{Open Access funding enabled and organized by CAUL and its Member Institutions}

Availability of data and materials The cryptocurrency data are collected from https://coinmarketcap.com/.

\section{Declarations}

Conflict of interest The authors declare that they have no competing interests.

Open Access This article is licensed under a Creative Commons Attribution 4.0 International License, which permits use, sharing, adaptation, distribution and reproduction in any medium or format, as long as you give appropriate credit to the original author(s) and the source, provide a link to the Creative Commons licence, and indicate if changes were made. The images or other third party material in this article are included in the article's Creative Commons licence, unless indicated otherwise in a credit line to the material. If material is not included in the article's Creative Commons licence and your intended use is not permitted by statutory regulation or exceeds the permitted use, you will need to obtain permission directly from the copyright holder. To view a copy of this licence, visit http:// creativecommons.org/licenses/by/4.0/. 


\section{References}

Allen, F., Gu, X., \& Jagtiani, J. (2021). A Survey of Fintech Research and Policy Discussion. Review of Corporate Finance, 1(3-4), 259-339

Baur, D. G., \& Hoang, L. T. (2020). A crypto safe haven against Bitcoin. Finance Research Letters. In Press.

Baur, D.G., Hong, K. \& Lee, A.D., (2018). Bitcoin: Medium of exchange or speculative assets? Journal of International Financial Markets, Institutions and Money 54, 177-189

Beigel, O. (2020). Who accepts bitcoins as payment? Available at https://99bitcoins.com/bitcoin/ who-accepts/

Bouri, E., Saeed, T., Vo, X. V., \& Roubaud, D. (2021). Quantile connectedness in the cryptocurrency market. Journal of International Financial Markets, Institutions and Money, 71, 101302

Bollen, B., \& Inder, B. (2002). Estimating daily volatility in financial markets utilizing intraday data. Journal of Empirical Finance, 9(5), 551-562

Bullmann, D., Klemm, J., \& Pinna, A. (2019). search for stability in crypto-assets: are stablecoins the solution? Occasional Paper Series 230, European Central Bank

Demir, E., Gozgor, G., Lau, C. K. M., \& Vigne, S. A. (2018). Does economic policy uncertainty predict the Bitcoin returns? An empirical investigation. Finance Research Letters, 26, 145-149

Dyhrberg, A. H. (2016). Bitcoin, gold and the dollar-A GARCH volatility analysis. Finance Research Letters, 16, 85-92

Eichengreen, B. (2019). From Commodity to Fiat and Now to Crypto: What Does History Tell Us?, NBER Working Papers 25426, National Bureau of Economic Research, Inc

Ferreira, P., Kristoufek, L., \& Pereira, E. (2020). DCCA and DMCA correlations of cryptocurrency markets. Physica A, 545, 123803

Griffin, J. M., \& Shams, A. (2020).Is Bitcoin Really Untethered? The Journal of Finance, 75, 1913-1964

Grobys, K. (2021). When the blockchain does not block: On hackings and uncertainty in the cryptocurrency market. Quantitative Finance (forthcoming).

Hazlett, P. K., \& Luther, W. J. (2019). Is bitcoin money? And what that means. The Quarterly Review of Economics and Finance, 77, 144-149

Hairudin, A., Sifat, I. M., Mohamad, A., \& Yusof, Y. (2020). Cryptocurrencies: A survey on acceptance, governance and market dynamics. International Journal of Finance \& Economics , 1-27

Hoang, L. T., \& Baur, D. G., How Stable Are Stablecoins? (January 31, 2020). Available at SSRN: https:// ssrn.com/abstract $=3519225$ or https://doi.org/10.2139/ssrn. 3519225

de la Horra, L., de la Fuente, G., \& Perote, J. (2020). The Drivers of Bitcoin Demand: A Short and LongRun Analysis. International Review of Financial Analysis, 62, 21-34

Lyons, R. K., \& Viswanath-Natraj, G. (2020). What Keeps Stablecoins Stable? NBER Working Paper No. w27136, Available at SSRN: https://ssrn.com/abstract $=3597868$

Moratis, G. (2021). Quantifying the spillover effect in the cryptocurrency market. Finance Research Letters, 38,101524

Nguyen, V. H. T., Nguyen, T. B., Nguyen, K. S., \& Pham, H. (2019). Asymmetric monetary policy effects on cryptocurrency markets. Research in International Business and Finance, 48, 335-339

Nguyen, V. H. T., Nguyen, T. B., Nguyen, C. T., \& Nguyen, Q. Q. (2019). Bitcoin return: Impacts from the introduction of new altcoins. Research in International Business and Finance, 48, 420-425

Parkinson, M. (1980). The Extreme Value Method for Estimating the Variance of the Rate of Return. The Journal of Business, 53, 61-65

Philippi, S., Schuhmacher, M., \& Bastian, N. (2021). Attracting Investors in Initial Coin Offerings: The Relevance of Specific Technological Capabilities for Fundraising Success. Review of Corporate Finance, 1(3-4), 455-485

Smales, L. A. (2019). Bitcoin as a safe haven: Is it even worth considering? Finance Research Letters, 30, 385-393

Xu, Q., Zhang, Y., \& Zhang, Z. (2021). Tail-risk spillovers in cryptocurrency markets. Finance Research Letters, 38, 101453

Publisher's Note Springer Nature remains neutral with regard to jurisdictional claims in published maps and institutional affiliations. 